\title{
$9-2011$
}

\section{Moving Academic Department Functions to Social Networks and Clouds: Initial Experiences}

\author{
George K. Thiruvathukal \\ Loyola University Chicago, gkt@cs.luc.edu \\ Konstantin Läufer \\ Loyola University Chicago, klaeufer@gmail.com \\ David Dennis \\ Loyola University Chicago, ddennis@luc.edu
}

Follow this and additional works at: https://ecommons.luc.edu/cs_facpubs

Part of the Computer Sciences Commons

\section{Recommended Citation}

Konstantin Läufer, George K. Thiruvathukal, David Dennis, "Moving Academic Department Functions to Social Networks and Clouds: Initial Experiences," Computing in Science and Engineering, vol. 13, no. 5, pp. 84-89, Sep./Oct. 2011, doi:10.1109/MCSE.2011.85

This Article is brought to you for free and open access by the Faculty Publications and Other Works by Department at Loyola eCommons. It has been accepted for inclusion in Computer Science: Faculty Publications and Other Works by an authorized administrator of Loyola eCommons. For more information, please contact ecommons@luc.edu. cc) (†) $\Theta$

This work is licensed under a Creative Commons Attribution-Noncommercial-No Derivative Works 3.0 License. Copyright @ 2011 Konstantin Läufer, George K. Thiruvathukal, David Dennis 


\section{MoVIng ACADEMIC DePARTMENT FUNCTIONS TO SOCIAL NETWORKS AND ClOUdS: INITIAL EXPERIENCES}

\section{By Konstantin Läufer, George K. Thiruvathukal, and David Dennis}

cademic departments at colleges and universities perform various functions to foster collaboration among their community members and to expand their communities by attracting new students, faculty, and staff. To perform these functions as effectively as possible, departments choose processes and technologies that support these goals and are appropriate for their target user groups. Making these choices is easier when department members understand both the technologies themselves and the digital culture of their users.

As new technologies emerge and digital culture evolves, academic departments (and university IT support organizations in general) must decide which technologies to adopt and when to implement them to continue functioning effectively. Here, we report on our experiences with transitioning from conventional approaches to new approaches based on hosted social networking sites ${ }^{1}$ and other cloudbacked sites ${ }^{2}$ for three central department functions:

- course management by faculty members;

- research collaboration among faculty, students, and external collaborators; and

- engagement with the community served by a department-including the dissemination of departmental announcements-to enable students and alumni to network and interact.
Here, we describe the social and cultural context that informed our technology choices, ${ }^{3}$ as well as the evolution of the choices themselves. ${ }^{1} \mathrm{We}$ then identify the targeted department functions and their actors, and describe the past and present technical architectures used to support these functions.

\section{Social Context: Generations and Technology}

It's useful to consider technology within the social and cultural context in which it's used. In particular, making technology choices can benefit from understanding the digital culture of the actors involved.

Digital culture has evolved in four major stages across the post-WWII generations.

- Baby Boomers (1946-1964). This generation was raised with various types of print and broadcast media, and postal mail and landline telephones for communication. The Internet emerged as a mainstream technology only after this generation was well into adulthood.

- Generation X (1965-1981). This post-baby-boom generation was the first to have widespread access to television during their formative years. Mobile phones and the Internet emerged as a mainstream technology when members of this generation were in their teens or twenties.

- Generation Y (1982-1995). This generation was born in the later stages of the personal computing era and the early Internet, before broadband became widely available. Its members embraced mobile phones and social networking as teenagers or young adults.

- Generation Z (1996-present). This "Internet Generation of Digital Natives" is being born into a world of mobile smart phones connected through social networking.

The boundaries among generations are blurred, and many individuals fall into more than one generation in terms of their technology-related behavior and habits.

As we learned in our departments, engaging the different generations can be challenging. Baby boomers and even gen-Xers tend to be suspicious of new technologies as fads, whereas gen-Yers are more open, and gen-Zers appear to be fully digital in orientation. More importantly, gen-Yers and gen-Zers are increasingly willing to live more "public" private lives and are much more comfortable with sharing their personal data with others.

Given that generation $Y$ represents all current college students, and the digital natives of generation $Z$ are now entering college and will constitute the vast majority of users over the next 10 years, this cultural shift will have profound implications for the future of academic management processes.

\section{Evolving Interaction}

As we now describe, we underwent distinct transitions as we adopted 
online interaction technology to support the three key academic functions.

\section{The Distant Pre-Web Past}

The distant past, by Internet standards, refers to the pre-Web era. Although the Web had been in existence since 1989 in the form of hyperlinked text documents, it didn't evolve beyond this until the wider commercial adoption of the Web in the mid 1990s. During this period, various disjointed mechanisms were used to provide content in a read-only fashion, including FTP, Gopher, and Wide Area Information Server (WAIS).

This period also saw the development of various means of interaction, although these were likewise disassociated:

- Email enabled asynchronous communication with individuals.

- Usenet groups supported public and internal discussions.

- FTP uploads facilitated document submissions.

Accordingly, online interaction among the various academic department actors we've described was limited mostly to email and Usenet, and even that was possible only among the few people who had access to such mechanisms.

\section{The Recent Web and Email Past}

In the mid 1990s, commercial adoption of the Web increased tremendously and read-only sites consolidated content dissemination on the Web.

Interactive offerings included interactive discussion fora; first-generation, consumer-oriented websites for photo sharing, driving directions, and so on; and developer-oriented sites for collaborative development. Fueled by the dramatic growth in Internet

access among the general population, email skyrocketed as the main means of nonpublic interaction.

\section{The Social Networking Present}

The early 2000s saw the emergence of a phenomenon often called Web 2.0. The major change brought about by Web 2.0 has been to engage users in the creation and classification of content. Examples are communitybased content creation efforts such as Wikipedia and the widespread use of tags instead of preset categories.

Furthermore, while first-generation interactive websites have been designed as silos that required screen-scraping for programmatic interaction, most secondgeneration functionality is serviceoriented and exposes a documented API for programmatic interaction. ${ }^{4}$

\section{The major change brought about by Web 2.0 has}

\section{been to engage users in the creation and classification}

\section{of content.}

This change is technologically significant because it allows individual applications to focus on performing fewer functions better and supporting cross-integration.

The middle of this past decade gave birth to two related trends: social networking and cloud computing. In cloud terminology, social networks are typically software-as-a-service (SaaS) offerings. Based on the SaaS paradigm, a wide range of well-known, cloud-backed offerings have emerged:

- general social networking sites, such as Facebook and Orkut;

- specialized professional networking sites, such as LinkedIn and XING;
- hosted conventional and lightweight blogs, such as Blogger and Tumblr, respectively;

- microblogging sites such as Twitter;

- social bookmarking sites such as Delicious and StumbleUpon;

- Web content management sites such as Google Sites;

- online document management and groupware sites such as Google Docs and Zoho; and

- social coding sites such as Bitbucket and GitHub.

Most organizations/departments have experienced and are experiencing a similar evolution, even if not everyone is ready to take the "social plunge." We'll now discuss how we're putting modern Web technologies to work in our department/laboratory. 
- submission and evaluation of code examples,

- student presentations and their evaluation,

- test taking and evaluation,

- sharing grades with students, and

- conducting student polls.

The workflow for research collaboration among computer science faculty, students, and external collaborators involves the following activities:

- knowledge gathering,

- project planning,

- collaborative writing and software development,

- bibliography management,

- formal publication, and

- other types of dissemination.

\section{As teachers and researchers, faculty members belong}

\section{mostly to the baby boomer and $X$ generations.}

The community served by a department includes current students, faculty, and staff; prospective students; alumni (former students); employers; candidates for faculty or staff positions; as well as more loosely connected groups, such as current students' parents. The complex, amorphous function of community engagement comprises several areas, including

- maintaining the department's official public Web content;

- maintaining the department's internal content (intranet);

- facilitating the department's internal processes, such as faculty meetings and committee decisions;

- enabling faculty to maintain Web content specific to their teaching and research activities;
- disseminating various types of departmental announcements to current and former students;

- enabling networking among current students with each other and departmental faculty and staff;

- enabling alumni (former students) to network with each other and departmental faculty and staff; and

- disseminating entry-level job postings to current students and advanced job postings to alumni.

Our list, of course, isn't intended to be exhaustive; it's part of a living process that will require us to reevaluate the environment and technologies that can support it on an ongoing basis. We nevertheless believe that the general categories (course management,

research collaboration, and community) are likely to stay with us forever.

\section{Actors: The Who and What}

Various actors are involved in all of these workflows and scenarios.

As teachers and researchers, faculty members belong mostly to the baby boomer and X generations. As computer scientists and scholars exposed to university information technologies, they tend to be more technologysavvy than other members of their generations but still display some of their characteristics, such as privacy concerns and a reluctance to participate in social networking. Younger faculty members are being hired only very slowly in these departments.

Staff members in job-specific rolessuch as secretary, system administrator, and so on-are predominantly baby boomers and gen-Xers. They tend to understand the technology they use to perform their jobs, but in many cases require training-especially for upgrades or new systems.

Current students, undergraduate and graduate alike, almost overwhelmingly belong to generation Y. Most students are regular social networking users and have expectations that the organizations they interact with participate in social networking as well. Prospective students will overwhelmingly belong to generation $Z$ and beyond. They'll have even higher expectations of managing their interactions through social networking. In particular, they'll expect their initial engagement with academic institutions or departments to occur through social networking tools. ${ }^{5}$

Alumni (former students) span multiple generations and use social networking accordingly. They're particularly interested in networking for social reasons or in search of employment and will engage in technology that supports these goals. ${ }^{6}$ External users, such as employers, candidates for faculty or staff positions, and students' parents, are a highly diverse group in terms of age and education level. Nevertheless, as these individuals belong to more and more recent generations, their expectations of using social networking for their interactions with academic institutions or departments will continue to increase.

\section{Evolving Architecture}

In our efforts to support the three targeted department functions, our technical architecture has evolved along with the available technologies themselves.

\section{Course Management}

In the distant pre-Web past, little course management technology was 
available. Instructors provided printed copies of their course materials, collected homework and project submissions on paper and/or removable media (floppy disks), and posted grades on their office doors. Using email to interact with students was then considered an innovation.

In the more recent past, the central IT Services unit at our university acquired a major commercial course management system that was intended to provide all required functionality online. Although acceptance was widely encouraged and continual training has been provided, the system itself has been criticized by numerous faculty members for its inflexibility, poor usability, regular and enforced "course deletion" requirements (owing to space limits), and occasional data loss (often associated with the preceding).

At present, we've abandoned the central system in favor of a combination of several carefully chosen, free, partially integrated "best-of-breed" cloud-backed technologies:

- Course materials dissemination occurs via cloud-backed solutions such as Google Sites and hosted blogs.

- Group discussions take place in Google Groups or closed Facebook groups.

- Submission of code examples takes place through social coding sites such as Bitbucket, which provide shared private code repositories that support standard versioncontrol software such as Mercurial. In addition, these sites allow students to create a project wiki, set up an issue tracker, and so on.

- Written work is submitted through Google Docs.

- Gradebook functionality is provided through Google Docs spreadsheets.
We're currently working to implement a gradebook with support for various roles, including instructor, student, and teaching assistant.

- Some online test taking and polls are conducted using Google Forms.

Each of us has been using this approach on sites for our computer science and history courses at Loyola University Chicago.

\section{Research Collaboration}

In the distant past, research collaboration was largely ad hoc. Researchers often exchanged code and documents through email or removable media, and used early version-control systems such as Source Code Control

- Knowledge gathering occurs on project-specific Wikis hosted on Google Sites, which supports finegrained access control.

- Project planning takes place with the help of Basecamp, a simple, effective, and highly usable site.

- Collaborative software development is supported effectively by distributed version-control systems (DVCS) such as Git or Mercurial. Various social coding sites, such as GitHub and Bitbucket, support these DVCSs. We use Bitbucket for nonpublic development because of its free private repositories and GitHub for open-source projects because of its high visibility and social coding features.

\section{At present, numerous collaborative research efforts,}

\section{including our own, have transitioned to a combination}

\section{of cloud-backed technologies.}

System and Revision Control System only for more systematic collaborative document authoring and code development. Knowledge gathering and interaction took place in meetings, by email, phone, or Unix talk.

In the recent past, more modern version-control systems such as Concurrent Versions System and Subversion were adopted, often with Secure Shell-based setups that supported collaboration across institutions. Interaction remained largely the same, except that newer forms of instant messaging replaced Unix talk.

At present, numerous collaborative research efforts, including our own, have transitioned to a combination of cloud-backed technologies. Specifically, we use the following (free) systems:
- Collaborative writing is the first of two writing phases. In this phase, the authors use Google Docs until they have a coherent draft. Then they switch to an appropriate formal publication mechanism in the second phase (discussed later).

- Bibliography management can greatly benefit from sharing and centralization. We have identified CiteULike as the most effective option for sharing bibliographic information among collaborators and publicly.

- Before formal publication can occur, suitable venues must be identified. WikiCFP lets us manage a list of conferences and workshops to which we plan to submit. Twitter is also useful for receiving announcements from conferences, such as postponed submission deadlines. 
- Formal publication itself is the second of two writing phases. In this phase, we use LaTeX or LyX to satisfy the envisioned publisher's formatting requirements; versioncontrol systems, as described earlier, enable collaboration.

Other types of dissemination include announcing a paper's publication on a blog such as Tumblr, which can automatically send short post versions to Twitter; including it in our CiteULike bibliographies; and emailing it to specific individuals. Disqus is a discussion mechanism that integrates with Tumblr, among other sites. Gravatar is a service used by various other sites for mapping email addresses to avatar pictures. Finally, Google Analytics provides various types of website access data (including blogs), which are useful in estimating the impact of a project or publication.

\section{Community Engagement}

In the distant past, community engagement occurred mostly through conventional channels such as mailings, poster advertisements, on-campus meetings, and so on. Usenet groups also played a role in allowing interaction among members of an academic community.

In the recent past, universities attempted to move large parts of their conventional community engagement efforts to Web and email, with mixed results. For example, some departments maintained an official website and used Google Groups for reaching current students by email, but had no systematic way to reach alumni.

As to the present, our departments both established a social networking presence almost overnight by picking and integrating a few specific technologies. Our initial focus has been on engaging students and alumni, which in turn requires that students and alumni be allowed to participate actively and contribute. ${ }^{6,7}$

First, to engage our students, we created a Facebook page with a custom URL that's easy to remember. This page allows our current, former, and prospective students to interact with each other and department faculty informally. This choice was based on the observation that virtually every one of our students already had a Facebook account. We have also set up a Tumblr blog for all public departmental announcements, thereby decentralizing the content creation process among department faculty. All blog posts automatically feed into a departmental Twitter handle we set up. Discussions are enabled through Disqus. We're currently evaluating how to pipe the blog posts back into the existing Google-Groups-based email lists using cloud-backed technology.

Second, to engage our alumni, we created a LinkedIn group so as to underscore the professional nature of our effort. We were able to populate this group quickly based on departmental information and one of the authors' personal alumni contacts on LinkedIn and via email. We periodically ask the members of this group to spread the word to other alumni who are not yet members.

A lthough already successful, we continue to refine our cloudbacked course management approach in terms of both technology and process. Our cloud-backed approach to research collaboration has functioned effectively as well and continues to evolve as we evaluate new technologies. We believe that both approaches can be used now as starting points for other departments and research groups.

Our social-networking-based approach to community engagement has been well received and, according to Google Analytics, draws more traffic to the departmental websites. Thus far, our focus has been on connecting students and alumni, but we plan to extend it to include various other aspects of community engagement.

The related work we've surveyed is descriptive, ${ }^{8,9}$ focuses on one particular technology, ${ }^{10-12}$ or proposes to build an educational social networking site from scratch. ${ }^{13}$ By contrast, our approach focuses on integrating existing, mature "best-of-breed" sites.

To understand how our observations relate to government agencies' ongoing efforts to broaden participation in computing, we need further data on social networking participation across demographic aspects other than age, such as gender, ethnicity, and education level. In addition, further data on social networking participation across different countries ${ }^{14}$ would be useful for generalizing across national boundaries.

Finally, technology continues to evolve rapidly. Requirements also tend to evolve as the target users change with respect to demographics and technology use. Therefore, IT decision makers at various levels of an academic organization must collaborate closely on requirements and carefully evaluate the available choices. s:

\section{References}

1. A.C. Weaver and B.B. Morrison, "Social Networking," Computer, vol. 41, no. 2, 2008, pp. 97-100.

2. A. Lenk et al., "What's Inside the Cloud? An Architectural Map of the Cloud Landscape," Proc. 2009 ICSE Workshop on Software Engineering Challenges of Cloud Computing, IEEE CS Press, 2009; doi:10.1109/CLOUD.2009.5071529.

3. P. Brandtzæg and J. Heim, "Why People Use Social Networking Sites," Online 
Communities and Social Computing, vol. 5621, Springer, Berlin, 2009, pp. 143-152.

4. M. Weiss and G.R. Gangadharan, "Modeling the Mashup Ecosystem: Structure and Growth," R\&D Management, vol. 40, no. 1, 2010, pp. 40-49.

5. R. Lindbeck and B. Fodrey, "Using Technology in Undergraduate Admission: A Student Perspective," J. College Admission, vol. 208, 2010, pp. 11-17.

6. A. Goetchius, Career Building through Social Networking, Rosen Publications, 2008.

7. R.D. Waters et al., Engaging Stakeholders through Social Networking: How Nonprofit Organizations Are Using Facebook," Public Relations Review, vol. 35, no. 2, 2009, pp. 102-106.

8. L. Lockyer and J. Patterson, "Integrating Social Networking Technologies in Education: A Case Study of a Formal Learning Environment," Proc. Int'I Conf. Advanced Learning Technologies (ICALT), IEEE CS Press, 2008; doi:10.1109/ICALT.2008.67.

9. H.T. Hung and S.C. Yuen, "Educational Use of Social Networking Technology in Higher Education," Teaching in Higher Education, vol. 15, no. 6, 2010, pp. 703-714.
10. P. Ractham and D. Firpo, "Using Social Networking Technology to Enhance Learning in Higher Education: A Case Study Using Facebook," Proc. 44th Hawaii Int'I Conf. System Sciences (HICSS), IEEE Press, 2011, pp. 1-10.

11. M. Roblyer et al., "Findings on Facebook in Higher Education: A Comparison of College Faculty and Student Uses and Perceptions of Social Networking Sites," The Internet and Higher Education, vol. 13, no. 3, 2010, pp. 134-140.

12. N. Selwyn, "Faceworking: Exploring Students' Education-Related Use of Facebook," Learning, Media and Technology, vol. 34, no. 2, 2009, pp. 157-174.

13. G. Conole and J. Culver, "The Design of Cloudworks: Applying Social Networking Practice to Foster the Exchange of Learning and Teaching Ideas and Designs," Computers \& Education, vol. 54, no. 3, 2010, pp. 679-692.

14. A. Mislove et al., "Measurement and Analysis of Online Social Networks," Proc. 7th ACM Sigcomm Conf. Internet Measurement, ACM Press, 2007; doi:10.1145/1298306.1298311.
Konstantin Läufer is a professor of computer science at Loyola University Chicago. His research interests include programming languages, software architecture, and distributed and pervasive computing, with applications in bioinformatics, environmental science, and digital humanities. Läufer has a $\mathrm{PhD}$ in computer science from the Courant Institute at New York University. Contact him at laufer@cs.luc.edu.

George K. Thiruvathukal is a professor of computer science and codirector of the Center for Textual Studies/Digital Humanities at Loyola University Chicago. He is an associate editor in chief for CiSE and an associate editor for Computing Now. Contact him at gkt@ cs.luc.edu or visit his page at http://home. thiruvathukal.com.

David Dennis is a associate professor in the Department of History at Loyola University Chicago. His research interests include modern European intellectual and cultural history, modern German history, art history, music and history, and Beethoven studies. Dennis has a PhD in history from the University of California, Los Angeles. Contact him at dennis@ luc.edu.

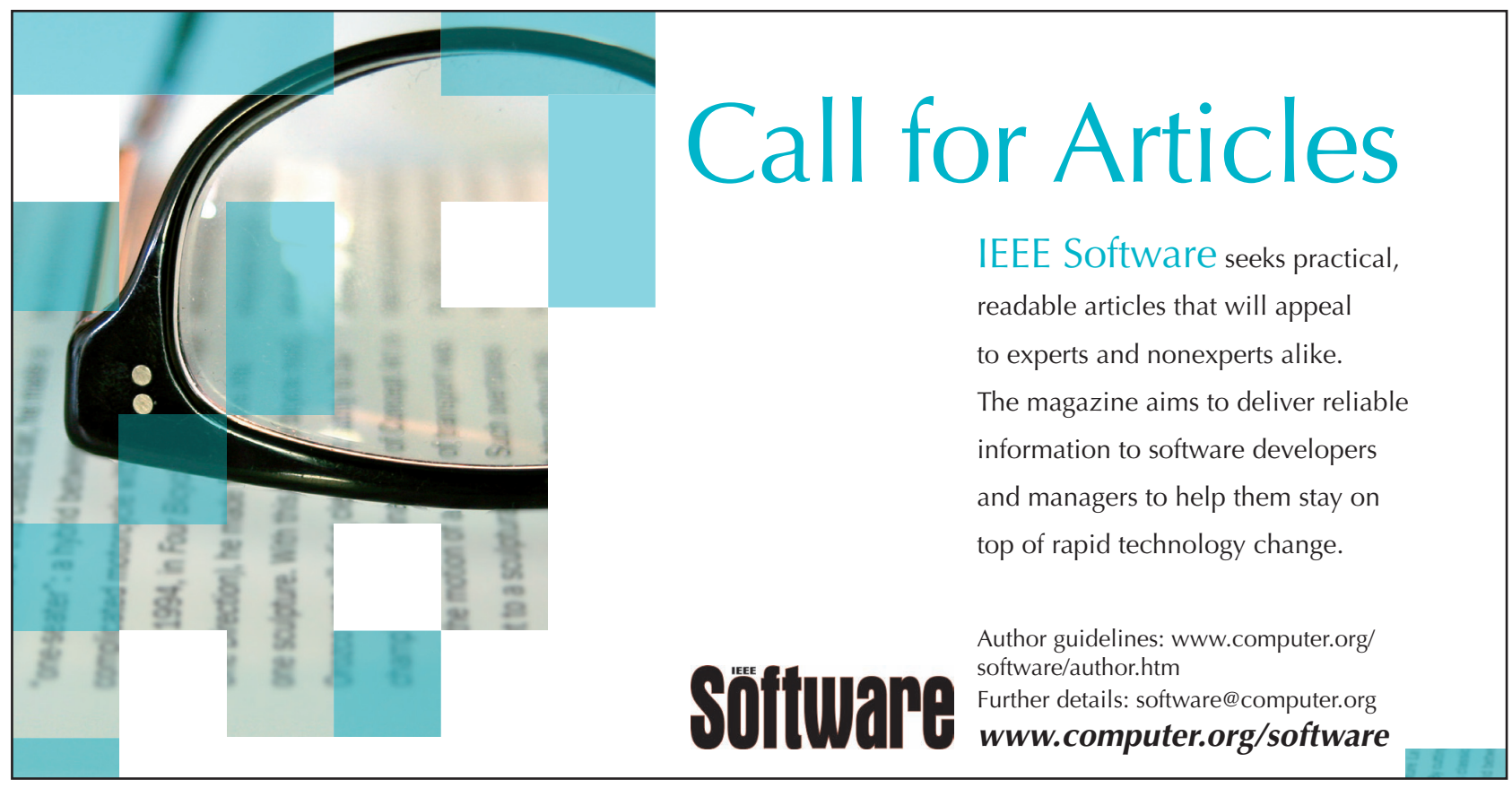

